\title{
Safe Storage of Sugar Bagasse
}

\author{
T.G. Myers ${ }^{1} \dagger$, and S.L. MitcheLL ${ }^{2}$ \\ ${ }^{1}$ Centre de Recerca Matemàtica, Barcelona \\ ${ }^{2}$ University of Limerick \\ (Communicated to MIIR on 23 June 2021)
}

Study Group: MISGSA2018. 16-20 January, 2017, African Institute for Mathematical Sciences.

Communicated by: David P. Mason

Industrial Partner: Sugar Milling Research Institute, Durban, South Africa

Presenter: Richard Loubser, Sugar Mill Research Institute

Team Members: H. Laurie, University of Cape Town; S.L. Mitchell, University of Limerick; T.G. Myers, Centre de Recerca Matemàtica, Barcelona.

Industrial Sector: Food and Drink

Tools: PDEs, Stefan problem

Key Words: Combustion, Food technology Sugar cane

MSC2020 Codes: 80

$\dagger$ Corresponding Author. tmyers@crm.cat 


\title{
SAFE STORAGE OF SUGAR BAGASSE
}

\author{
T. G. Myers*and S. L. Mitchell ${ }^{\dagger}$ \\ Study group participants
}

T.G. Myers, S.L Mitchell, H. Laurie

\begin{abstract}
In this report we investigate the thermal evolution in a one-dimensional bagasse stockpile. The mathematical model is taken from the work of Gray et al. [4] and involves four unknowns, the temperature, oxygen, liquid water and water vapour. We first non-dimensionalise the model to identify dominant terms and so simplify the system. We then calculate solutions for the approximate and full system. It is shown that under certain conditions spontaneous combustion will occur. Most importantly we show that spontaneous combustion can be avoided by sequential building. For example, in a situation where say a $6 \mathrm{~m}$ stockpile can spontaneously combust we could construct a $3 \mathrm{~m}$ pile then some days later add another $3 \mathrm{~m}$ to produce a stable $6 \mathrm{~m}$ pile.
\end{abstract}

\section{Introduction}

Bagasse is the residue which remains after sugar cane has been crushed and the majority of the juice extracted. In the past it was viewed as a waste product, however it is now used as a biofuel and also in the manufacture of building materials. Outside of South Africa bagasse has been used as a fuel in the factory boilers for co-generation of steam and electricity. This obviously reduces costs and so improves competitiveness. Unfortunately, it is well-known that large piles of bagasse are prone to spontaneous combustion.

The Sugar Milling Research Institute (SMRI) situated in KwaZulu-Natal in South Africa is interested in storing bagasse for use in their furnaces, but due to obvious safety issues they would first like to understand the processes behind spontaneous combustion. Consequently, at the 2016 and 2017 Mathematics in Industry Study Group meetings the SMRI discussed three issues related to safe storage of bagasse:

\footnotetext{
*Centre de Recerca Matemàtica, Campus de Bellaterra, Edifici C, 08193 Bellaterra, Barcelona, Spain. email: tmyers@crm.cat

${ }^{\dagger}$ MACSI, Department of Mathematics and Statistics, University of Limerick, Limerick, Ireland. email: sarah.mitchell@ul.ie
} 
(i) Calculating the maximum height of the bagasse heap required to avoid spontaneous combustion;

(ii) Investigating whether or not there are advantages in adjusting the moisture content;

(iii) Investigating whether or not there is an advantage in pelletizing the bagasse.

The first recorded bagasse spontaneous combustion incident took place in the Mourilyan stockpile in 1983. This incident motivated experiments, some of which were made in $[1,2]$ in 1984, that attempted to find out why bagasse would spontaneously combust and which conditions led to this phenomenon. Following two more ignition incidents between 1983 and 1988, Dixon [3] investigated the process of spontaneous combustion of bagasse and found that moisture content plays a very significant role in the process. Recommendations were therefore made that the effect of moisture content should never be neglected in the mathematical modelling of the spontaneous combustion of bagasse stockpiles.

Spontaneous combustion has been observed in a number of other industries and consequently there is a rich literature on the topic. In the following we base our work on the one-dimensional models developed by Gray and co-workers [1, 2, 3, 4].

\section{Governing equations}

Our mathematical study is based on the following four equations:

$$
\begin{aligned}
\left(\rho_{b} c_{b}+m_{w} c_{w} W\right) \frac{\partial T}{\partial t}=k \frac{\partial^{2} T}{\partial x^{2}} & +q_{d} \rho_{b} z_{d} X \exp \left(-\frac{E_{d}}{R T}\right)+q_{w} \rho_{b} z_{w} W X \exp \left(-\frac{E_{w}}{R T}\right) g(T) \\
& +L_{v}\left[z_{c} V-z_{e} W \exp \left(-\frac{L_{v}}{R T}\right)\right] \\
\frac{\partial V}{\partial t}= & D_{V} \frac{\partial^{2} V}{\partial x^{2}}+z_{e} W \exp \left(-\frac{L_{v}}{R T}\right)-z_{c} V \\
\frac{\partial W}{\partial t}= & -z_{e} W \exp \left(-\frac{L_{v}}{R T}\right)+z_{c} V \\
\frac{\partial X}{\partial t} & =D_{X} \frac{\partial^{2} X}{\partial x^{2}}-f \rho_{b} z_{d} X \exp \left(-\frac{E_{d}}{R T}\right)-f \rho_{b} z_{w} W X \exp \left(-\frac{E_{w}}{R T}\right) g(T)
\end{aligned}
$$

These represent conservation of energy via temperature $T(\mathrm{~K})$, water vapour $V\left(\mathrm{~mol} / \mathrm{m}^{3}\right)$, liquid water $W\left(\mathrm{~mol} / \mathrm{m}^{3}\right)$, and oxygen $X\left(\mathrm{~mol} / \mathrm{m}^{3}\right)$ (note we use a more natural notation for the variables than that prescribed in [4]). For the present study a one-dimensional model is considered therefore the quantities temperature, water vapour, liquid content and oxygen vary with the height of the stockpile, $\mathrm{x}$, and time, t. The exponential terms represent the heat sources and take a standard Arrhenius form, where $E_{d}$ is the activation energy for the dry reaction, $E_{w}$ for the wet reaction and $L_{v}$ the latent heat of vaporisation. The various other constants and typical values are described in Table 1. 
The function $g(T)$ is a switch that turns off the wet reaction at $T=T_{s}$,

$$
g(T)=\frac{1}{2}\left[\tanh \left(0.6\left(T_{s}-T\right)\right)+1\right] .
$$

The system is subject to the following boundary conditions. At the top surface, $x=L$, we impose Newton cooling conditions, stating that the exchange of temperature, vapour and oxygen are driven by the difference between the value on surrounding air and in the pile:

$$
-k \frac{\partial T}{\partial x}=h\left(T-T_{a}\right), \quad-D_{V} \frac{\partial V}{\partial x}=h_{V}\left(V-V_{a}\right), \quad-D_{X} \frac{\partial X}{\partial x}=h_{X}\left(X-X_{a}\right) .
$$

Assuming the bagasse is placed on an impermeable surface we impose the following at $x=0$,

$$
T=T_{a}, \quad \frac{\partial V}{\partial x}=0, \quad \frac{\partial X}{\partial x}=0 .
$$

These conditions are based on the assumption that the substrate prevents vapour and water from passing through. Since the temperature variation is so slow, on the order of days, it is assumed that it does not significantly affect the (infinitely large) substrate temperature and the temperatures match there. Obviously these conditions could be altered if more precise details of the storage area were provided.

We assume that the pile is initially well mixed so that all values are constant (and known)

$$
T(x, 0)=T_{i n}, \quad V(x, 0)=V_{i n}, \quad W(x, 0)=W_{i n}, \quad X(x, 0)=X_{i n} .
$$

To simplify the problem, we will now write the equations in non-dimensional form. The scaling is as follows:

$$
x^{\prime}=\frac{x}{L}, \quad t^{\prime}=\frac{t}{\Delta t}, \quad T^{\prime}=\frac{T}{T_{s}}, \quad V^{\prime}=\frac{V}{V_{a}}, \quad W^{\prime}=\frac{W}{\Delta W}, \quad X^{\prime}=\frac{X}{X_{a}},
$$

where the time- and water-scales, $\Delta t$ and $\Delta W$, are the only ones not yet specified.

Writing equation (1) in non-dimensional form, and immediately dropping the primes, gives

$$
\begin{gathered}
\left(\rho_{b} c_{b}+m_{w} c_{w} \Delta W W\right) \frac{T_{s}}{\Delta t} \frac{\partial T}{\partial t}=\frac{k T_{s}}{L^{2}} \frac{\partial^{2} T}{\partial x^{2}}+q_{d} \rho_{b} z_{d} X_{a} X \exp \left(-\frac{E_{d}}{R T_{s} T}\right) \\
+q_{w} \rho_{b} z_{w} \Delta W X_{a} W X \exp \left(-\frac{E_{w}}{R T_{s} T}\right) g\left(T_{s} T\right) \\
+L_{v}\left[z_{c} V_{a} V-z_{e} \Delta W W \exp \left(-\frac{L_{v}}{R T_{s} T}\right)\right] .
\end{gathered}
$$




\begin{tabular}{|c|c|c|c|}
\hline Parameter & Description & Value & Unit \\
\hline$E_{d}$ & activation energy of dry reaction & $1.08 \times 10^{5}$ & $\mathrm{~J} \mathrm{~mol}^{-1}$ \\
\hline$E_{w}$ & activation energy of wet reaction & $6.5 \times 10^{4}$ & $\mathrm{~J} \mathrm{~mol}^{-1}$ \\
\hline$R$ & universal gas constant & 8.31 & $\mathrm{~J} \mathrm{~mol}^{-1} \mathrm{~K}^{-1}$ \\
\hline$q_{d}$ & exothermicity of dry reaction & $1.7 \times 10^{7}$ & $\mathrm{~J} \mathrm{~kg}^{-1}$ \\
\hline$q_{w}$ & exothermicity of wet reaction & $6.6 \times 10^{6}$ & $\mathrm{~J} \mathrm{~kg}^{-1}$ \\
\hline$z_{d}$ & pre-exponential factor of the dry reaction & $2.7 \times 10^{7}$ & $\mathrm{~m}^{3} \mathrm{~mol}^{-1} \mathrm{~s}^{-1}$ \\
\hline$z_{w}$ & pre-exponential factor of the wet reaction & 0.204 & $\mathrm{~m}^{3} \mathrm{~mol}^{-1} \mathrm{~s}^{-1}$ \\
\hline$z_{e}$ & pre-exponential factor of evaporation & $3.41 \times 10^{4}$ & $\mathrm{~s}^{-1}$ \\
\hline$z_{c}$ & pre-exponential factor of condensation & 4.7 & $\mathrm{~s}^{-1}$ \\
\hline$L_{v}$ & latent heat of vaporisation & $4.2 \times 10^{4}$ & $\mathrm{~J} \mathrm{~mol}^{-1}$ \\
\hline$k$ & thermal conductivity & 0.5 & $\mathrm{~J} \mathrm{~m}^{-1} \mathrm{~K}^{-1} \mathrm{~s}^{-1}$ \\
\hline$D_{V}, D_{X}$ & water vapour and oxygen diffusivities, resp., & $2.5 \times 10^{-5}$ & $\mathrm{~m}^{2} \mathrm{~s}^{-1}$ \\
\hline & heat transfer coefficient & 5 & $\mathrm{~J} \mathrm{~m}^{-2} \mathrm{~K}^{-1} \mathrm{~s}^{-1}$ \\
\hline$h_{V}, h_{X}$ & water vapour and oxygen transfer coefficients, resp., & 5 & $\mathrm{~m} \mathrm{~s}^{-1}$ \\
\hline$m_{w}$ & mass of water & $1.8 \times 10^{-2}$ & $\mathrm{~kg} \mathrm{~mol}{ }^{-1}$ \\
\hline$\rho_{w}$ & density of water & $1 \times 10^{3}$ & $\mathrm{~kg} \mathrm{~m}^{-3}$ \\
\hline$\rho_{b}$ & density of dry bagasse & 125 & $\mathrm{~kg} \mathrm{~m}^{-3}$ \\
\hline$c_{w}$ & specific heat capacity of water & $4.19 \times 10^{3}$ & $\mathrm{~J} \mathrm{~kg}^{-1} \mathrm{~K}^{-1}$ \\
\hline$c_{b}$ & specific heat capacity of dry bagasse & $1.4 \times 10^{3}$ & $\mathrm{~J} \mathrm{~kg}^{-1} \mathrm{~K}^{-1}$ \\
\hline$T_{s}$ & switch temperature & 333 & $\mathrm{~K}$ \\
\hline$T_{a}$ & ambient bagasse temperature & 303 & $\mathrm{~K}$ \\
\hline$V_{a}$ & ambient water vapour concentration & 1.74 & $\mathrm{~mol} \mathrm{~m} \mathrm{~m}^{-3}$ \\
\hline$X_{a}$ & oxygen concentration in air & 8.04 & $\mathrm{~mol} \mathrm{~m}^{-3}$ \\
\hline$f$ & moles of $\mathrm{O}_{2}$ consumed per $\mathrm{kg}$ of bagasse & 33.33 & $\mathrm{~mol} \mathrm{~kg}{ }^{-1}$ \\
\hline$L$ & height of the bagasse pile & $3-6$ & $\mathrm{~m}$ \\
\hline
\end{tabular}

Table 1: Parameter values for computations 
The quantity of primary interest in this work is the temperature, and so we will work on the time scale of thermal diffusion. Balancing the left hand side with the first term on the right hand side leads to

$$
\Delta t=\frac{L^{2}\left(\rho_{b} c_{b}+m_{w} c_{w} \Delta W\right)}{k}=\frac{L^{2}}{D_{T}}, \quad D_{T}=\frac{k}{\left(\rho_{b} c_{b}+m_{w} c_{w} \Delta W\right)} .
$$

Taking values from Table 1 we obtain $\Delta t=4.56 \times 10^{6}$ s, i.e., approximately 53 days.

The three remaining equations (2)-(4) are now

$$
\begin{aligned}
\frac{V_{a}}{\Delta t} \frac{\partial V}{\partial t} & =\frac{D_{V} V_{a}}{L^{2}} \frac{\partial^{2} V}{\partial x^{2}}+z_{e} \Delta W W \exp \left(-\frac{L_{v}}{R T_{s} T}\right)-z_{c} V_{a} V \\
\frac{\Delta W}{\Delta t} \frac{\partial W}{\partial t}= & -z_{e} \Delta W W \exp \left(-\frac{L_{v}}{R T_{s} T}\right)+z_{c} V_{a} V \\
\frac{X_{a}}{\Delta t} \frac{\partial X}{\partial t}= & \frac{D_{X} X_{a}}{L^{2}} \frac{\partial^{2} X}{\partial x^{2}}-f \rho_{b} z_{d} X_{a} X \exp \left(-\frac{E_{d}}{R T_{s} T}\right) \\
& -f \rho_{b} z_{w} \Delta W X_{a} W X \exp \left(-\frac{E_{w}}{R T_{s} T}\right) g\left(T_{s} T\right)
\end{aligned}
$$

We can re-arrange (13) as

$$
\frac{1}{z_{e} \Delta t} \frac{\partial W}{\partial t}=-W \exp \left(-\frac{L_{v}}{R T_{s}}\right) \exp \left[\frac{L_{v}}{R T_{s}}\left(1-\frac{1}{T}\right)\right]+\frac{z_{c} V_{a}}{z_{e} \Delta W} V
$$

and so we may choose a value for $\Delta W$ by balancing terms on the right hand side. This gives

$$
\Delta W=\frac{z_{c} V_{a}}{z_{e}} \exp \left(\frac{L_{v}}{R T_{s}}\right)
$$

and, using values from Table 1 , we find $\Delta W=1.03 \times 10^{3} \approx 20$ minutes. So the water equilibrates much faster than the heat. Then (15) simplifies to

$$
\exp \left(\frac{L_{v}}{R T_{s}}\right) \frac{1}{z_{e} \Delta t} \frac{\partial W}{\partial t}=-W \exp \left[-\frac{L_{v}}{R T_{s}}\left(\frac{1}{T}-1\right)\right]+V
$$

The rapid equilibration of the water is reflected in the coefficient of the time derivative in (17),

$$
\frac{1}{Z_{e} \Delta t} \exp \left(\frac{L_{v}}{R T_{s}}\right) \sim 2.76 \times 10^{-5}
$$

This small value indicates that on the thermal time-scale the water equation is approximately steady-state. 
The full four-equation model, $(10,12,14,15)$ is now

$$
\begin{gathered}
\left(A_{0}+\bar{A}_{0} W\right) \frac{\partial T}{\partial t}=\frac{\partial^{2} T}{\partial x^{2}}+A_{1} X \exp \left[-\gamma_{d}\left(\frac{1}{T}-1\right)\right]+A_{2} W X \exp \left[-\gamma_{w}\left(\frac{1}{T}-1\right)\right] g(T) \\
+A_{3}\left\{V-W \exp \left[-\gamma_{v}\left(\frac{1}{T}-1\right)\right]\right\} \\
B_{0} \frac{\partial V}{\partial t}=\frac{\partial^{2} V}{\partial x^{2}}+B_{1}\left\{W \exp \left[-\gamma_{v}\left(\frac{1}{T}-1\right)\right]-V\right\}, \\
C_{0} \frac{\partial W}{\partial t}=-W \exp \left[-\gamma_{v}\left(\frac{1}{T}-1\right)\right]+V, \\
D_{0} \frac{\partial X}{\partial t}=\frac{\partial^{2} X}{\partial x^{2}}-D_{1} X \exp \left[-\gamma_{d}\left(\frac{1}{T}-1\right)\right]-D_{2} W X \exp \left[-\gamma_{w}\left(\frac{1}{T}-1\right)\right] g(T) .(21)
\end{gathered}
$$

The dimensionless parameters are

$$
\begin{aligned}
& A_{0}=\frac{\rho_{b} c_{b}}{\rho_{b} c_{b}+m_{w} c_{w} \Delta W}, \quad \bar{A}_{0}=\frac{m_{w} c_{w} \Delta W}{\rho_{b} c_{b}+m_{w} c_{w} \Delta W}, \\
& A_{1}=\frac{q_{d} \rho_{b} z_{d} X_{a} L^{2}}{k T_{s}} \exp \left(-\gamma_{d}\right), \quad A_{2}=\frac{q_{w} \rho_{b} z_{w} \Delta W X_{a} L^{2}}{k T_{s}} \exp \left(-\gamma_{w}\right), \quad A_{3}=\frac{L_{v} z_{c} V_{a} L^{2}}{k T_{s}}, \\
& B_{0}=\frac{L^{2}}{\Delta t D_{V}}, \quad B_{1}=\frac{z_{c} L^{2}}{D_{V}}, \quad C_{0}=\frac{\exp \left(\gamma_{v}\right)}{z_{e} \Delta t}, \\
& D_{0}=\frac{L^{2}}{\Delta t D_{X}}, \quad D_{1}=\frac{f \rho_{b} z_{d} L^{2}}{D_{X}} \exp \left(-\gamma_{d}\right), \quad D_{2}=\frac{f \rho_{b} z_{w} \Delta W L^{2}}{D_{X}} \exp \left(-\gamma_{w}\right) \\
& \gamma_{d}=\frac{E_{d}}{R T_{s}}, \quad \gamma_{w}=\frac{E_{w}}{R T_{s}}, \quad \gamma_{v}=\frac{L_{v}}{R T_{s}},
\end{aligned}
$$

and

$$
g(T)=\frac{1}{2}\left[\tanh \left(0.6 T_{s}(1-T)\right)+1\right] .
$$

Finally, the boundary conditions from (6) become

$$
-\frac{\partial T}{\partial x}=\alpha_{T}\left(T-T_{a} / T_{s}\right), \quad-\frac{\partial V}{\partial x}=\alpha_{V}(V-1), \quad-\frac{\partial X}{\partial x}=\alpha_{X}(X-1),
$$

on $x=1$, where

$$
\alpha_{T}=\frac{h L}{k}, \quad \alpha_{V}=\frac{h_{V} L}{D_{V}}, \quad \alpha_{X}=\frac{h_{X} L}{D_{X}} .
$$


On $x=0$ the boundary conditions (7) are simply

$$
T=\frac{T_{a}}{T_{s}}, \quad \frac{\partial V}{\partial x}=0, \quad \frac{\partial X}{\partial x}=0
$$

and the initial conditions (8) are

$$
T(x, 0)=\frac{T_{i n}}{T_{s}}, \quad V(x, 0)=\frac{V_{i n}}{V_{a}}, \quad W(x, 0)=\frac{W_{i n}}{\Delta W}, \quad X(x, 0)=\frac{X_{i n}}{X_{a}} .
$$

From Table 1 the values of these coefficients are (using $L=3 \mathrm{~m}$ ),

$$
\begin{gathered}
A_{0} \sim 0.69, \quad \bar{A}_{0} \sim 0.31, \quad A_{1} \sim 0.22, \quad A_{2} \sim 4.12, \quad A_{3} \sim 1.87 \times 10^{4} \\
B_{0} \sim 7.92 \times 10^{-2}, \quad B_{1} \sim 1.69 \times 10^{6}, \quad C_{0} \sim 2.76 \times 10^{-5} \\
D_{0} \sim 0.079, \quad D_{1} \sim 0.36, \quad D_{2} \sim 17.14 \\
\gamma_{d} \sim 36.3, \quad \gamma_{w} \sim 23.6, \quad \gamma_{v} \sim 15.3, \quad \alpha_{T} \sim 30, \quad \alpha_{V} \sim 6 \times 10^{5}, \quad \alpha_{X} \sim 6 \times 10^{5} .
\end{gathered}
$$

\section{Simplified model}

The coefficient $C_{0} \approx 2.8 \times 10^{-5}$ in front of the time derivative in (20) is very small and so a simplified model can be prescribed. Ignoring this term in (20) allows us to express $W$ in terms of $V$ and $T$ (or $V$ in terms of $W$ and $T$ ),

$$
W=V \exp \left[\gamma_{v}\left(\frac{1}{T}-1\right)\right]
$$

This will not satisfy the initial condition but since the time derivative is of $\mathcal{O}\left(10^{-5}\right)$ it should be a good approximation after a very short time period. This then permits a significant reduction of the vapour equation (19) to

$$
B_{0} \frac{\partial V}{\partial t}=\frac{\partial^{2} V}{\partial x^{2}}
$$

If we assume that $V_{i n}=V_{a}$ then the exact solution to this problem is $V=1$. If $V_{i n} \neq V_{a}$ then we can make use of the fact that the coefficient of the time derivative in (31) is $B_{0} \sim 0.08 \ll 1$. If we seek a perturbation solution for $V=V^{0}+\epsilon V^{1}+\cdots$, where $\epsilon=D_{T} / D_{V}$, we obtain $V^{0}=1, V^{n}=0$ for all $n>1$, and so we may assume $V=1$ to a high order of accuracy. Physically this tells us that vapour diffuses rapidly around the pile (compared to thermal diffusion).

The steady-state approximation for $W$ means that the final term in (18) disappears. Then we can write equation (18) for the temperature $T$ as

$$
\left(A_{0}+\bar{A}_{0} W\right) \frac{\partial T}{\partial t}=\frac{\partial^{2} T}{\partial x^{2}}+A_{1} X \exp \left[-\gamma_{d}\left(\frac{1}{T}-1\right)\right]+A_{2} W X \exp \left[-\gamma_{w}\left(\frac{1}{T}-1\right)\right] g(T) .
$$




\section{Numerical solution}

We briefly describe the numerical solution used to provide the qualitative and approximate results. We begin by writing (18)-(21) as

$$
\begin{aligned}
\left(A_{0}+\bar{A}_{0} W\right) \frac{\partial T}{\partial t} & =\frac{\partial^{2} T}{\partial x^{2}}+F_{T}(T, V, W, X), \\
B_{0} \frac{\partial V}{\partial t} & =\frac{\partial^{2} V}{\partial x^{2}}+F_{V}(T, V, W), \\
C_{0} \frac{\partial W}{\partial t} & =F_{W}(T, V, W), \\
D_{0} \frac{\partial X}{\partial t} & =\frac{\partial^{2} X}{\partial x^{2}}+F_{X}(T, V, W, X),
\end{aligned}
$$

and solve this system using the method of lines. We define the spatial mesh $x_{i}=i \Delta x$, for $i=0,1, \ldots, I$, where $\Delta x=1 / I$, and let $T_{i}=T\left(x_{i}, t\right)$, and similarly for $V_{i}, W_{i}$ and $X_{i}$. Then we discretise the diffusion terms in (33), (34) and (36) as

$$
\begin{aligned}
\left(A_{0}+\bar{A}_{0} W_{i}\right) \frac{\mathrm{d} T_{i}}{\mathrm{~d} t} & =\frac{T_{i+1}-2 T_{i}+T_{i-1}}{\Delta x^{2}}+F_{T}\left(T_{i}, V_{i}, W_{i}, X_{i}\right), \\
B_{0} \frac{\mathrm{d} V_{i}}{\mathrm{~d} t} & =\frac{V_{i+1}-2 V_{i}+V_{i-1}}{\Delta x^{2}}+F_{V}\left(T_{i}, V_{i}, W_{i}\right), \\
D_{0} \frac{\mathrm{d} X_{i}}{\mathrm{~d} t} & =\frac{X_{i+1}-2 X_{i}+X_{i-1}}{\Delta x^{2}}+F_{X}\left(T_{i}, V_{i}, W_{i}, X_{i}\right),
\end{aligned}
$$

for $i=1,2, \ldots, I-1$. Equation (35) is simply

$$
C_{0} \frac{\mathrm{d} W_{i}}{\mathrm{~d} t}=F_{W}\left(T_{i}, V_{i}, W_{i}\right)
$$

which holds for $i=0,1, \ldots, I$. The first boundary condition in (24) on $x=0$ immediately gives $T_{0}=T_{i n} / T_{s}$. For the other remaining conditions in (24), and those in (22) on $x=1$, we obtain further ODEs involving $V_{0}, X_{0}, T_{I}, V_{I}$ and $X_{I}$. These are

$$
\begin{aligned}
B_{0} \frac{\mathrm{d} V_{0}}{\mathrm{~d} t} & =\frac{2\left(V_{1}-V_{0}\right)}{\Delta x^{2}}+F_{V}\left(T_{0}, V_{0}, W_{0}\right), \\
D_{0} \frac{\mathrm{d} X_{0}}{\mathrm{~d} t} & =\frac{2\left(X_{1}-X_{0}\right)}{\Delta x^{2}}+F_{X}\left(T_{0}, V_{0}, W_{0}, X_{0}\right), \\
\left(A_{0}+\bar{A}_{0} W_{I}\right) \frac{\mathrm{d} T_{I}}{\mathrm{~d} t} & =\frac{2\left(T_{I-1}-T_{I}-\Delta x \alpha_{T}\left(T_{I}-T_{a} / T_{s}\right)\right)}{\Delta x^{2}}+F_{T}\left(T_{I}, V_{I}, W_{I}, X_{I}\right), \\
B_{0} \frac{\mathrm{d} V_{I}}{\mathrm{~d} t} & =\frac{2\left(V_{I-1}-V_{I}-\Delta x \alpha_{V}\left(V_{I}-1\right)\right)}{\Delta x^{2}}+F_{V}\left(T_{I}, V_{I}, W_{I}\right), \\
D_{0} \frac{\mathrm{d} X_{I}}{\mathrm{~d} t} & =\frac{2\left(X_{I-1}-X_{I}-\Delta x \alpha_{X}\left(X_{I}-1\right)\right)}{\Delta x^{2}}+F_{X}\left(T_{I}, V_{I}, W_{I}, X_{I}\right) .
\end{aligned}
$$

This system of ODEs is solved, with the initial conditions in (25), using ode23s in Matlab. 


\section{Results}

In Figure 1 we show the dimensional temperature and vapour distribution in a $3 \mathrm{~m}$ pile after approximately 46 days. Given our focus on spontaneous combustion, the temperature is the quantity of primary interest. From Figure 1a we can see that, so far, this pile gives no indication of thermal runaway. The initial constant temperature, $T_{i n}=65^{\circ} \mathrm{C}$ very quickly reduces to a curved profile satisfying the fixed temperature condition $T=30^{\circ} \mathrm{C}$ at $x=0$. The maximum temperature remains just below $60^{\circ} \mathrm{C}$ for almost the whole time period. After the initial transient the water vapour, shown in Figures 1b, remains close to $V_{a}\left(=1.74 \mathrm{~mol} \mathrm{~m}^{-3}\right)$ for the top two metres (as suggested by the simplified model) but decreases to just below 0.7 in the bottom metre. Liquid water, shown in Figure $2 \mathrm{a}$

(a)

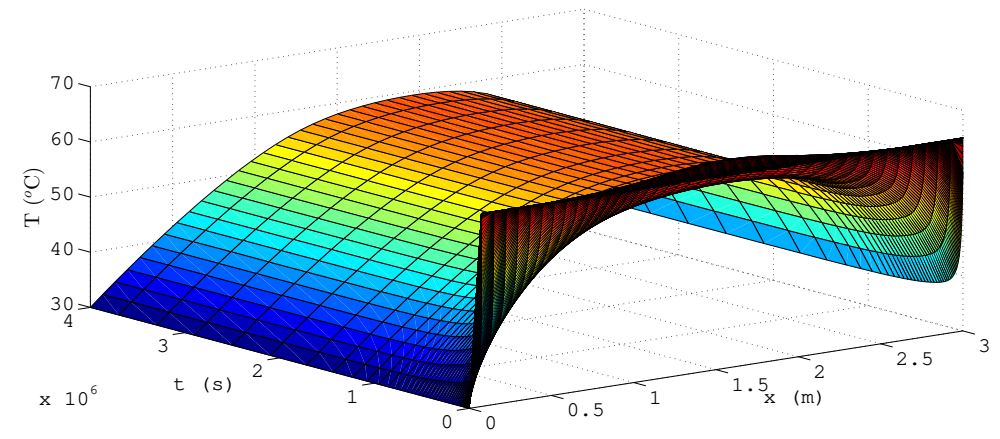

(b)

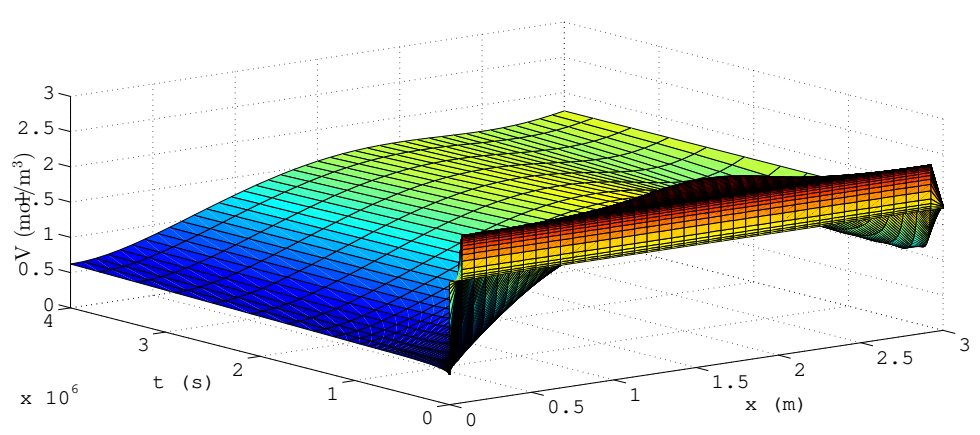

Figure 1: (a) Temperature in $3 \mathrm{~m}$ pile after $4 \times 10^{6} \mathrm{~s} \approx 46$ days. (b) Corresponding vapour distribution.

builds up at the substrate, where it cannot escape, and also at the top surface where presumably vapour from the air arrives and condenses to water. Finally, Figure 2b shows the oxygen content, which obviously decreases away from the top surface as it is required for the thermal reaction. 
(a)

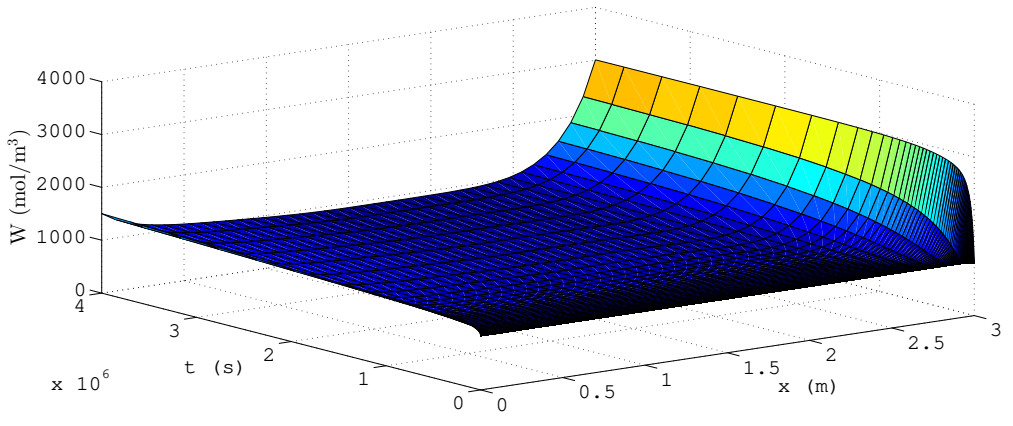

(b)

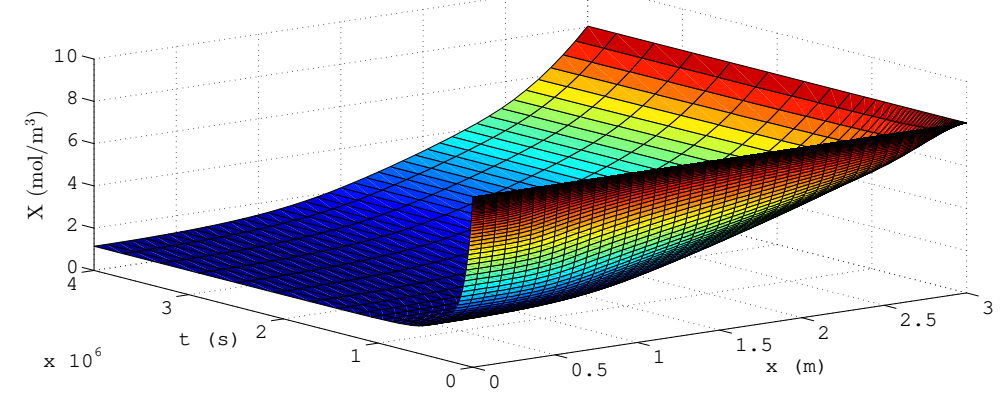

Figure 2: (a) Water distribution in $3 \mathrm{~m}$ pile. (b) Oxygen distribution.

In Figures 3a, b we present the temperature and oxygen profiles predicted by the simplified two equation model. Comparison with Figure 1a and 2b show excellent agreement, indicating that the simpler model can provide accurate results. This is slightly surprising, since the reduction is based on the assumption that $V$ is approximately constant, in dimensional form $V=V_{a}$. Yet the solution of the full model, Figure 1b shows clearly that $V$ is only close to the ambient value in the top $2 \mathrm{~m}$ of the pile. In the bottom metre it reduces by almost a factor 3. A possible reason why this error does not affect the temperature is that heat is primarily generated away from the bottom region, as we will see later, it is generated where the oxygen level is high, i.e., near the surface. Consequently, although the vapour approximation is inaccurate near the substrate the fact that it is accurate where heat is generated results in a good approximation of the overall temperature profile.

In Figure 5a we show an example where the pile is close to thermal runaway. The pile is $5 \mathrm{~m}$ high and the temperature is plotted up to 46 days. It is interesting to note the evolution of the temperature. After the initial transient from a well mixed pile the profile resembles that of the pile shown in Figure 1a for a long time but the maximum is slowly rising: taking approximately 36 days to increase by ten degrees. In the final five days there is a rise of 60 degrees, reaching a final maximum of over $150{ }^{\circ} \mathrm{C}$. In Figure $5 \mathrm{~b}$ we show the temperature one day later, now the maximum is close to $180^{\circ} \mathrm{C}$ the point where the maximum occurs has moved towards the surface, since the oxygen content lower down in the pile is close to zero. If we continue the simulation much longer the program crashes, 
(a)

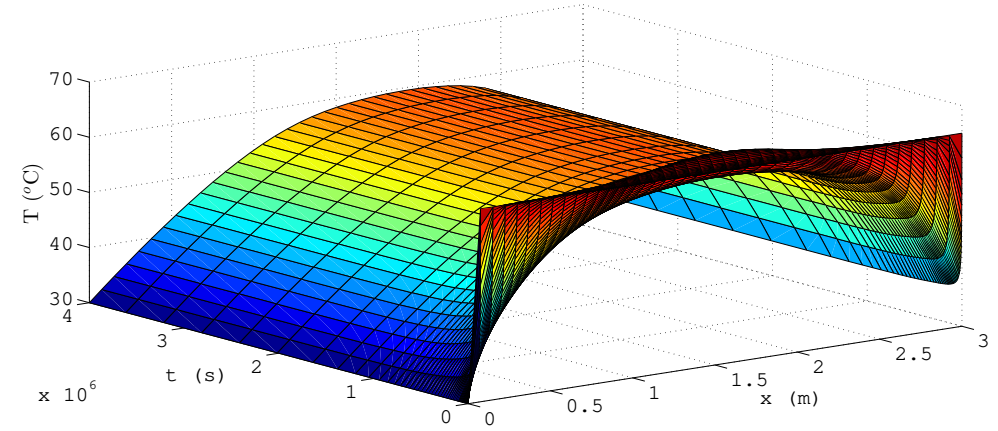

(b)

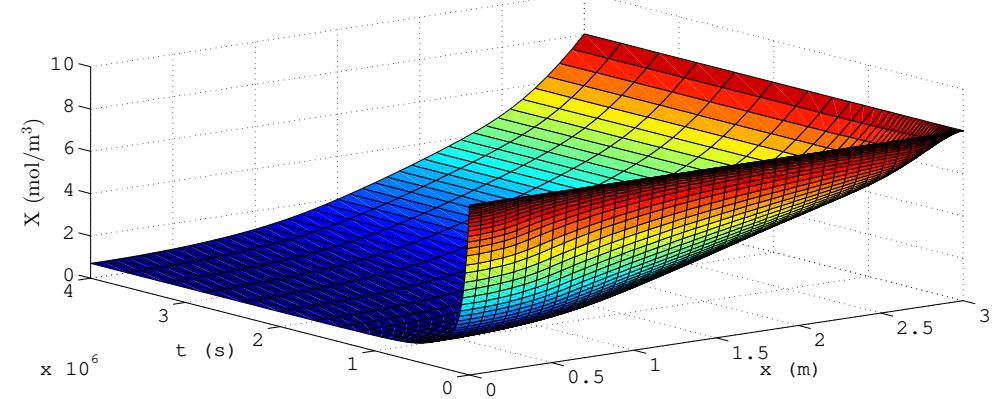

Figure 3: Results from two equation model. (a) Temperature in $3 \mathrm{~m}$ pile after $4 \times 10^{6} \mathrm{~s} \approx 46$ days. (b) Corresponding oxygen distribution. 
(a)

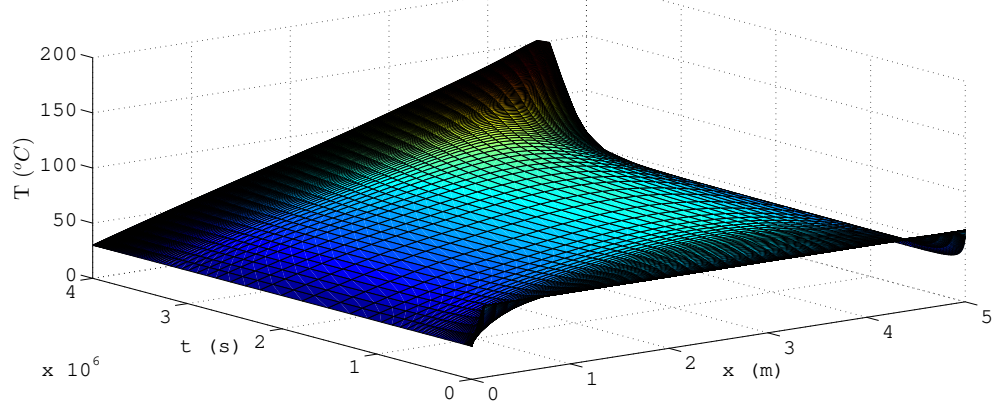

(b)

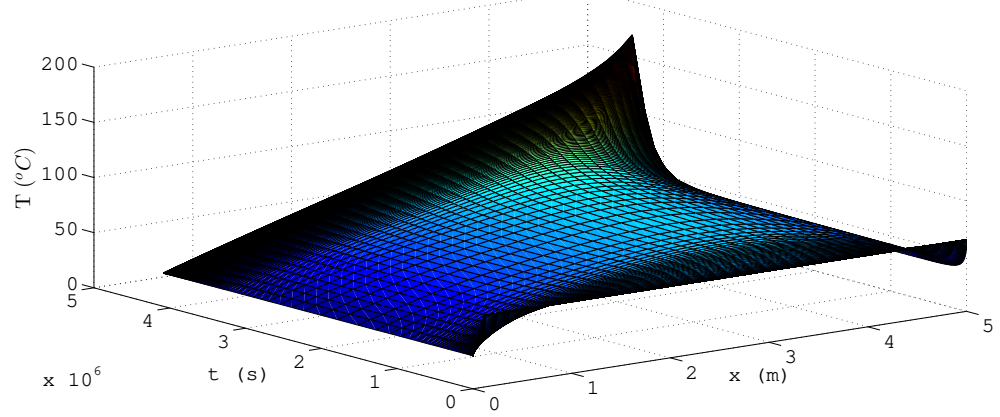

Figure 4: Temperature $T$ in a $5 \mathrm{~m}$ high pile after, (a) $4 \times 10^{6} \mathrm{~s}$ (approximately 46 days), (b) 47 days. 
due to a rapid increase in temperature.

Finally, in Figures 5a, b we demonstrate a possible way forward in the storage of combustible materials. Figure 5a shows the temperature distribution in a $3 \mathrm{~m}$ pile one day after being laid down. This demonstrates how rapidly the temperature adjusts to the environment. At this stage we add another two metres of material, so the initial condition for Figure 5b is the temperature in the $3 \mathrm{~m}$ pile after one day and then a constant temperature in the top two metres. Again we see a rapid adjustment but, significantly, after 46 days the maximum temperature is just above 60 degrees and showing no signs of thermal runaway. In fact, the temperature is slowly decreasing as heat loss to the surroundings is stronger than the heat generation. This is in contrast to the $5 \mathrm{~m}$ pile formed in a single stage where the maximum is almost $150{ }^{\circ} \mathrm{C}$ and increasing rapidly. If we run the same simulation with the two equation model then the temperature profile takes the same form. The maximum temperature, around $68^{\circ}, \mathrm{C}$ is reached after 8 days and the minimum, at the end of the simulation is approximately $64{ }^{\circ} \mathrm{C}$. The four equation model reaches its maximum of $67{ }^{\circ} \mathrm{C}$ more rapidly, taking close to 4.6 days and the final minimum is $63{ }^{\circ} \mathrm{C}$. This indicates that the simpler, two equation model can provide accurate results with less computation (however, further tests reveal that the two equation model will predict runaway at earlier times than the full model).

(a)

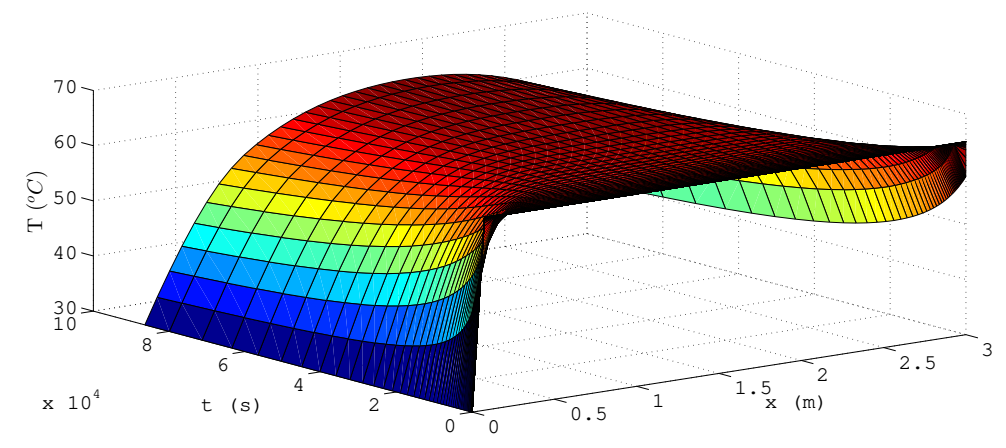

(b)

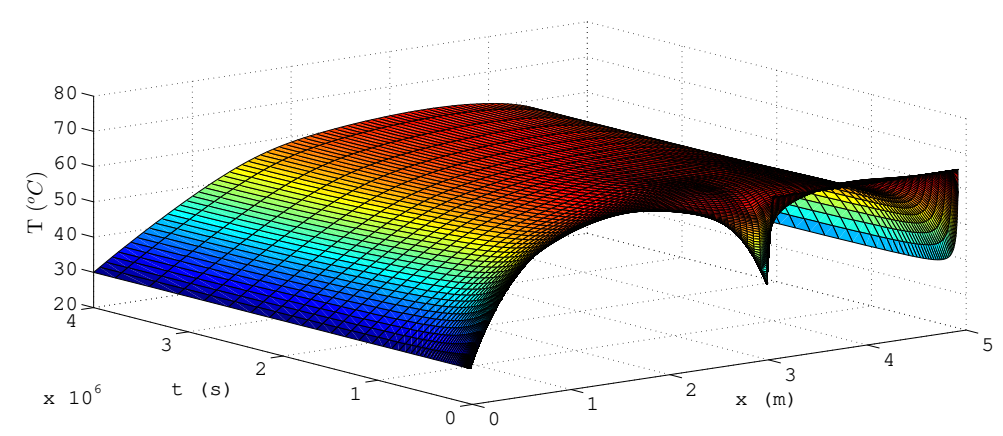

Figure 5: Temperature in (a) a $3 \mathrm{~m}$ pile after 1 day, (b) a $5 \mathrm{~m}$ pile after 46 days (made by adding another $2 \mathrm{~m}$ to the $3 \mathrm{~m}$ pile after 1 day) 


\section{Conclusion}

In this report we have presented a mathematical model, coupling the effects of temperature, liquid water, water vapour and oxygen. The numerical solution of this system shows that it can predict thermal runaway. Significantly, it also shows a strategy for building large piles without leading to ignition. Specifically, if we build a pile sequentially we can avoid spontaneous combustion. In our calculations we demonstrated this with the example of a single $5 \mathrm{~m}$ pile, which reached ignition temperature after around 47 days. However, if instead we laid down a $3 \mathrm{~m}$ pile, left it for only a day and then added another $2 \mathrm{~m}$ there was no sign of combustion. Of course we could continue this strategy to make larger piles, with more layers, but with this single example we have paved the way for a more complete investigation.

The simplified system, where the vapour was set to its ambient value and the liquid water expressed by a simple analytical expression, led to good agreement with the full model prior to thermal runaway, although tests did show that the two equation model would predict runaway at earlier times.

This brings us to the main question proposed by the SMRI: how high can a bagasse pile be built without spontaneous combustion occurring? The answer depends crucially on the method of building. A $5 \mathrm{~m}$ pile laid down in a single go could ignite, whereas a pile made up of a $3 \mathrm{~m}$ one followed by a $2 \mathrm{~m}$ one may not, even if there is only a single day between adding the second layer. Multiple layer piles could be significantly higher than single layer piles. So, there is no simple answer to the question posed by the SMRI. However, there is a clear recommendation: build sequentially, to allow the initial heat to escape resulting in thermally stable piles.

\section{References}

[1] B.F. Gray. Progress report to Sugar Milling Research Institute, University of KwaZuluNatal, Durban, July 1984.

[2] B.F. Gray, J.F. Griffiths, S.M. Hasko. Spontaneous ignition hazards in stockpiles of cellulosic materials: criteria for safe storage. J. Chem. Tech. and Biotech A. (1984), 34,453 .

[3] T.F. Dixon. Spontaneous combustion in bagasse stockpiles. Proceedings of the 1988 Conference of the Australian Society of Sugar Cane Technologists, Cairns, Queensland, Australia. Edited by B.T. Egan, 1988, pp. 53 - 61.

[4] B.F. Gray, M.J. Sexton, B. Halliburton, C. Macaskill. Wetting-induced ignition in cellulosic materials. Fire Safety J. (2002), 37, 465 - 479.

[5] B.W. Halliburton. Investigation of spontaneous combustion phenomenology of bagasse and calcium hypochlorite. Ph.D. thesis, Macquarie University, Sydney, Australia, 2001. 